\title{
Static and kinetic friction force and surface roughness of different archwire- bracket sliding contacts
}

\author{
Francisco J CARRION-VILCHES ${ }^{1}$, María-Dolores BERMUDEZ ${ }^{1}$ and Paula FRUCTUOSO² \\ ${ }^{1}$ Grupo de Ciencia de Materiales e Ingeniería Metalúrgica, Universidad Politécnica de Cartagena. C/Doctor Fleming sn. 30202 Cartagena, Spain \\ ${ }^{2}$ Centros de Estudios Vitaldent, C/Albala, 5. 28037 Madrid, Spain \\ Corresponding author, Francisco J CARRION-VILCHES; E-mail: fjc.vilches@upct.es
}

\begin{abstract}
The aim of this study was to determine the static and kinetic friction forces of the contact bracket-archwire with different dental material compositions in order to select those materials with lower resistance to sliding. We carried out sliding friction tests by means of a universal testing machine following an experimental procedure as described in ASTM D1894 standard. We determined the static and kinetic friction forces under dry and lubricating conditions using an artificial saliva solution at $36.5^{\circ} \mathrm{C}$. The bracket-archwire pairs studied were: stainless steel-stainless steel; stainless steel-glass fiber composite; stainless steel-Nitinol 60; sapphire-stainless steel; sapphire-glass fiber composite; and sapphire-Nitinol 60. The best performance is obtained for Nitinol 60 archwire sliding against a stainless steel bracket, both under dry and lubricated conditions. These results are in agreement with the low surface roughness of Nitinol 60 with respect to the glass fiber composite archwire. The results described here contribute to establishing selection criteria for materials for dental archwire-brackets.
\end{abstract}

Keywords: Static and kinetic force, Nitinol 60, Glass fiber composite, Surface roughness

\section{INTRODUCTION}

The operation of binding a bracket to a guiding archwire generates friction at the archwire-bracket interface ${ }^{1}$. During orthodontic treatment, the contact with the bracket also leads to surface damage and wear of the archwire material ${ }^{2}$. Static frictional force (the force needed to start the relative sliding of two surfaces) inhibits the movement of the tooth. When the elastically deformed wire uprights the tooth, friction is reduced and movement starts. Kinetic frictional force appears as the tooth moves in the direction of the applied force.

The use of materials with low friction coefficients reduces anchorage strain. In fact, it has been estimated that friction between bracket and archwire can cause up to a $50 \%$ loss of force ${ }^{2}$.

Over the past years, a great number of new orthodontic materials and manufacturing techniques have been developed and used as alternatives to the classical stainless steel wires and brackets. However, friction forces must be determined for each new combination in order to predict their performance when they are used in clinical applications. This need has led to research interest in this field; as the substitution of classical materials such as stainless steel by the new aesthetic ceramic brackets, requires new research efforts in to assess the possible advantages and disadvantages of each type of wire-bracket contact.

In order to compare the friction performance of different materials for the bracket/archwire contact, it is necessary to consider a number of variables. Firstly, it is important to take into account the different friction values obtained as a function of the medium, i.e. dry or lubricated conditions, in the presence of artificial saliva. The surface finish of the materials and roughness values are a critical variable in determining friction values; it is expected that the smoothest surface will give the lowest friction.

Yu et al. ${ }^{3,4)}$ found that stainless steel wire exhibited the smoothest surface topography, when compared with the titanium alloys.

Choi et $a l . .^{5}$ found significantly lower surface roughness values and higher friction values for ceramic brackets sliding against archwire, while the glass or filler-reinforced polymer presented higher static friction values, as compared with the conventional stainless steel control bracket.

The recent work by Murayama et al. ${ }^{6)}$ recommended using a wire with the smallest possible roughness for displacements longer than $1.0 \mathrm{~mm}$. They also found that the friction force of a martensite Ni-Ti wire was significantly greater than that of other aesthetic and austenitic wires.

Chang et $a l .^{7)}$ have reported an increase of friction force in the presence of saliva. Studying friction between wire and bracket in artificial saliva, Fidalgo et al. ${ }^{8}$ ) found that the combination between ceramic brackets and stainless steel wire showed a high friction coefficient when compared with the combination of stainless steel wire against polymer (polycarbonate) bracket.

In the present work, we have determined surface roughness, static and kinetic friction forces both under dry sliding conditions and in the presence of artificial saliva at body temperature. Six bracket-archwire combinations have been studied, by using stainless steel (SS) or sapphire (SP) brackets against stainless steel (SS), glass fiber composite (GF) or Nitinol 60 (Niti) wires. 


\section{MATERIALS AND METHODS}

Brackets, archwires and ligature

Two different edgewise brackets were tested, one made of ferritic stainless steel (SS) from World Class Technology and the other was made of sapphire (SP) from BioMers. Three archwires were used in the tests: Nitinol (Niti) from TP Orthodontics; translucent archwire (GF) from BioMers; and austenitic stainless steel (SS) archwire from TP orthodontics. The elastomeric ligature used in all cases was from TP orthodontics. The archwires, brackets and elastomeric ligature used in this study correspond to commercial products frequently used in dental practice. Details of all materials are referred to in Table 1.

Roughness of the surfaces of all materials was measured using a non-contact 3D profiler from Taylor Hobson. The measurements of the surface roughness (Table 2) are shown using the mean roughness depth $(\mathrm{Rz})$, the root mean square roughness $(\mathrm{Rq})$ and average roughness ( $\mathrm{Ra}$ ) that is the arithmetic mean value of the roughness profile ordinates, which is one of the most effective surface roughness measures commonly adopted in general engineering practice.

The samples were observed using a Hitachi S-3500N scanning electronic microscope (SEM); in the case of the sapphire (SP) bracket and translucent archwire (GF) the samples were coated with gold.

A universal testing machine from Hounsfield equipped with a load cell of $50 \mathrm{~N}$ (with an accuracy of $0.002 \mathrm{~N}$ ) was used to obtain the friction force data in all the tests. Figure 1 shows the testing machine with bracket-archwire assembly.

The friction results obtained correspond to the mean values after five repetitions of each case tested. Data relating to the static forces were obtained from the first maximum peak in the initial stage of the tests. Thereafter, the data correspond to kinetic forces, corresponding to mean values of the stationary stage of the graphs.

The test conditions in both dry and lubricated configuration employed a velocity of $5 \mathrm{~mm} / \mathrm{min}$ with a load weight attached of $1.50 \mathrm{~N}$ and a distance travelled by the archwire of $10 \mathrm{~mm}$. The tests were conducted at room temperature under a relative humidity of $50 \%$ for the dry and the wet tests, using an artificial saliva solution from Kin Hidrat Gel which was at $36.5^{\circ} \mathrm{C}$. The tests were carried out under a relative humidity of $50 \%$ at room temperature.

Table 2 shows the arithmetic mean, standard deviation and variance of the friction tests for every archwire-bracket pair tested under dry or lubricated conditions.

\section{RESULTS}

Figures 2 and 3 show the friction-distance plots for the different configurations: SP (Fig. 2) or SS (Fig. 3)

Table 1 List of components used and related information

\begin{tabular}{cccc}
\hline Component & Material & Product and nominal size & Manufacturer \\
\hline \multirow{3}{*}{ Archwire } & Glass Fiber Composite & Translucent Fiberglass $(0.0190 \times 025 ")$ & BioMers \\
& Austenitic Staniless Steel & Rounded Rectangular $(0.019 \times 0.025 ")$ & TP Orthodontics \\
& Ni/60/Ti/40 & Rounded Rectangular (0.019×0.025") & TP Orthodontics \\
Bracket & Ferritic Stainless Steel & Ortho Classic (Slot dimension 0.022") & World Class Technology \\
& Sapphire & Aesthetic MBT (Slot dimension 0.022") & BioMers \\
Ligature & Elastomer & Mini Stix low friction & TP Orthodontics \\
\hline
\end{tabular}

Table 2 Data of the static and kinetic force in the archwire-bracket pairs

\begin{tabular}{|c|c|c|c|c|c|c|c|c|c|c|c|c|c|c|c|c|c|c|c|}
\hline \multicolumn{4}{|c|}{ Bracket } & \multicolumn{4}{|c|}{ Archwire } & \multicolumn{6}{|c|}{ Static Friction Force, N } & \multicolumn{6}{|c|}{ Kinetic Friction Force, N } \\
\hline \multirow{2}{*}{ Material } & \multicolumn{3}{|c|}{ Roughness, $\mu \mathrm{m}$} & \multirow{2}{*}{ Material } & \multicolumn{3}{|c|}{ Roughness, $\mu \mathrm{m}$} & \multicolumn{3}{|c|}{ Dry } & \multicolumn{3}{|c|}{ Lubricated } & \multicolumn{3}{|c|}{ Dry } & \multicolumn{3}{|c|}{ Lubricated } \\
\hline & $\mathrm{R}_{\mathrm{a}}$ & $\mathrm{R}_{\mathrm{q}}$ & $\mathrm{R}_{\mathrm{z}}$ & & $\mathrm{R}_{\mathrm{a}}$ & $\mathrm{R}_{\mathrm{q}}$ & $\mathrm{R}_{\mathrm{z}}$ & $\bar{x}$ & $\sigma$ & $\sigma^{2}$ & $\bar{x}$ & $\sigma$ & $\sigma^{2}$ & $\bar{x}$ & $\sigma$ & $\sigma^{2}$ & $\bar{x}$ & $\sigma$ & $\sigma^{2}$ \\
\hline \multirow{3}{*}{ Sapphire } & \multirow{3}{*}{0.559} & \multirow{3}{*}{0.754} & \multirow{3}{*}{5.072} & Nitinol & 0.145 & 0.268 & 1.747 & 3.35 & 0.83 & 0.69 & 2.79 & 0.99 & 0.98 & 3.44 & 0.83 & 0.69 & 2.33 & 0.88 & 0.77 \\
\hline & & & & Stainless Steel & 0.111 & 0.161 & 1.040 & 6.87 & 1.28 & 1.64 & 4.74 & 0.41 & 0.17 & 4.65 & 1.11 & 1.23 & 3.27 & 0.50 & 0.25 \\
\hline & & & & GF Composite & 0.659 & 0.817 & 5.196 & 4.12 & 0.10 & 0.01 & 4.13 & 0.28 & 0.78 & 4.13 & 0.28 & 0.08 & 3.02 & 1.50 & 2.25 \\
\hline \multirow{3}{*}{ Stainless Steel } & \multirow{3}{*}{0.350} & \multirow{3}{*}{0.405} & \multirow{3}{*}{2.564} & Nitinol & 0.145 & 0.268 & 1.747 & 2.37 & 0.64 & 0.41 & 2.84 & 0.81 & 0.66 & 2.33 & 0.58 & 0.34 & 2.99 & 0.86 & 0.74 \\
\hline & & & & Stainless Steel & 0.111 & 0.161 & 1.040 & 5.09 & 0.11 & 0.01 & 5.58 & 0.36 & 0.13 & 5.05 & 0.10 & 0.01 & 5.50 & 0.35 & 0.12 \\
\hline & & & & GF Composite & 0.659 & 0.817 & 5.196 & 4.45 & 0.40 & 0.16 & 3.12 & 0.82 & 0.67 & 3.87 & 0.44 & 0.19 & 3.00 & 0.76 & 0.58 \\
\hline
\end{tabular}

$\bar{x}$ : Arimetic Mean, $\sigma$ : Standard deviation, $\sigma^{2}$ : Variance 


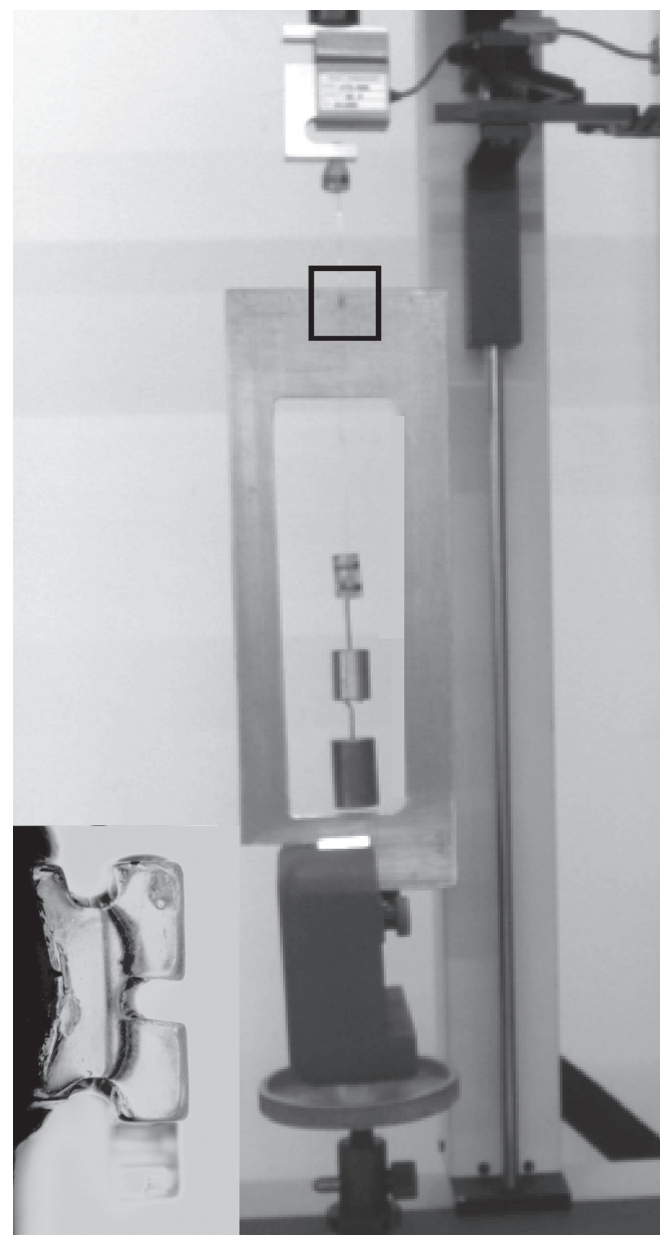

Fig. 1 Test machine and close-up of bracket-archwire assembly.

brackets against Niti, SS or GF archwires; under two conditions: dry and lubricated at $36.5^{\circ} \mathrm{C}$.

The data show the static and kinetic friction forces, as a result of the analysis of the friction force obtained as described in the experimental section. If we focus on the static friction data as being the main factor for the resistance to start the movement, the high values obtained when the SS archwire was tested should be highlighted. In both cases, dry or lubricated, and using brackets of SP or SS, the values are the highest in this study, with 6.87 and $5.58 \mathrm{~N}$, respectively.

The best results and the lowest values of static friction are found with the use of Niti archwire in all test configurations. The pair Niti-SS is particularly noteworthy, giving a value of static friction of $2.37 \mathrm{~N}$ in dry condition.

In Fig. 4 we have plotted a 3D graph of the roughness values for the archwires and brackets against the static friction force. It can be seen that the lubricated tests present the best results.
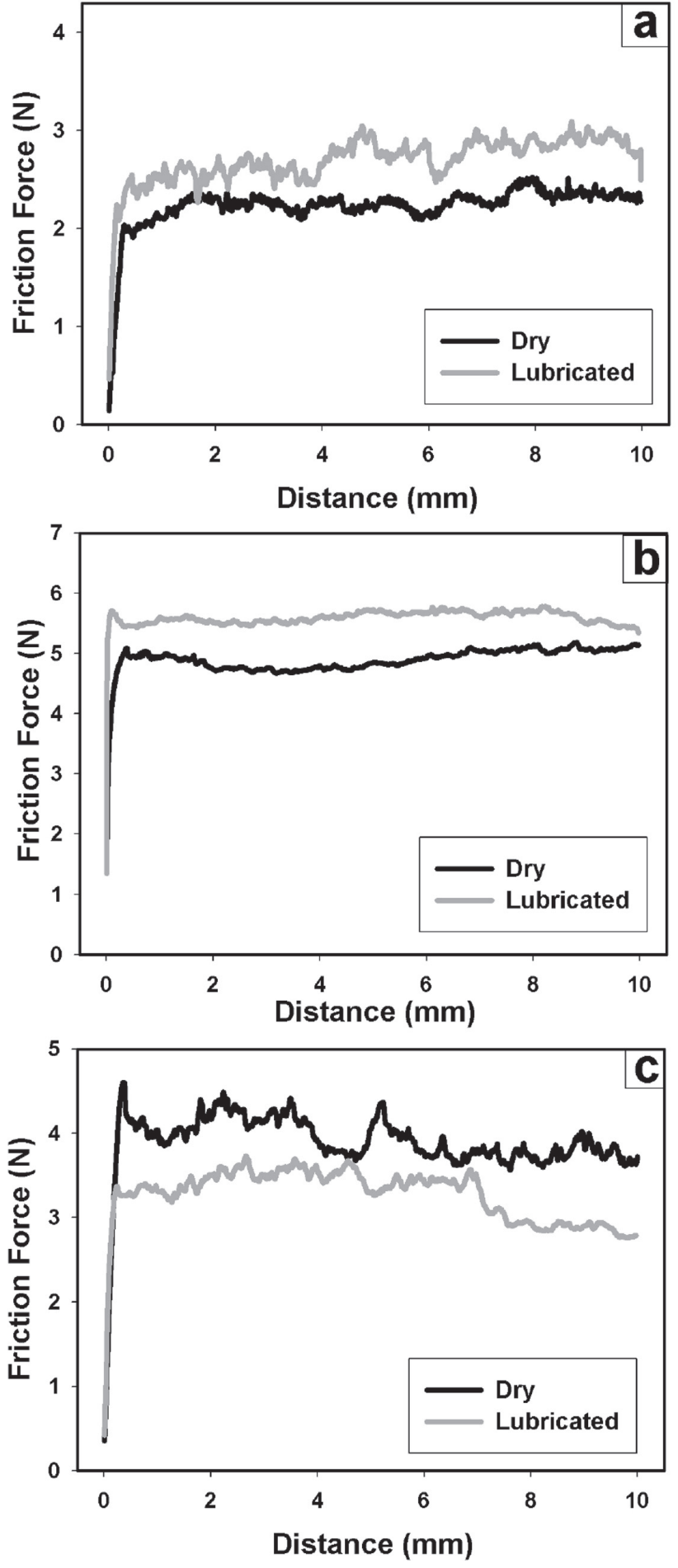

Fig. 2 Friction force plots of the sapphire bracket against: a-Nitinol, b-Stainless Steel and c-glassfiber composite archwire under dry and lubricated contact. 

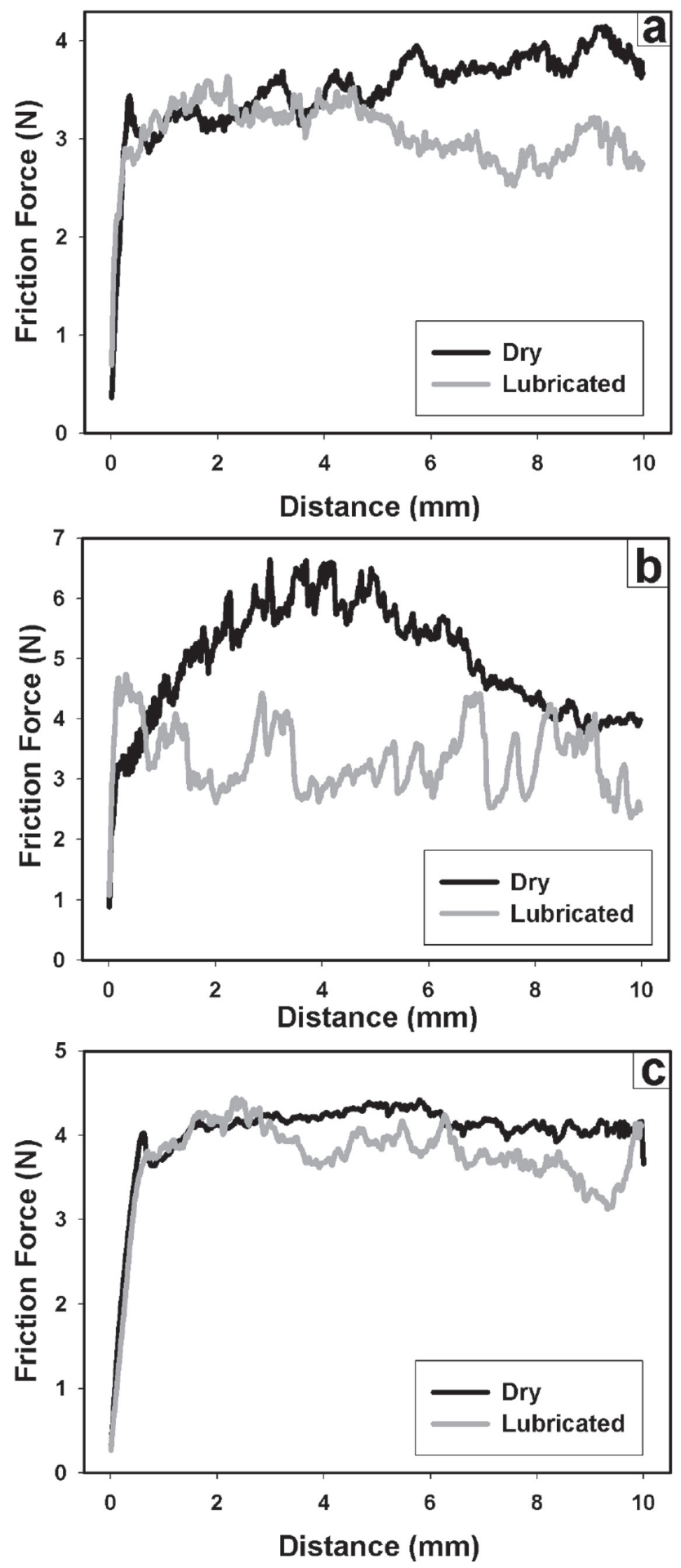

Fig. 3 Friction force plots of the Stainless Steel bracket against: a-Nitinol, b-Stainless Steel and c-glassfiber composite archwire under dry and lubricated contact.

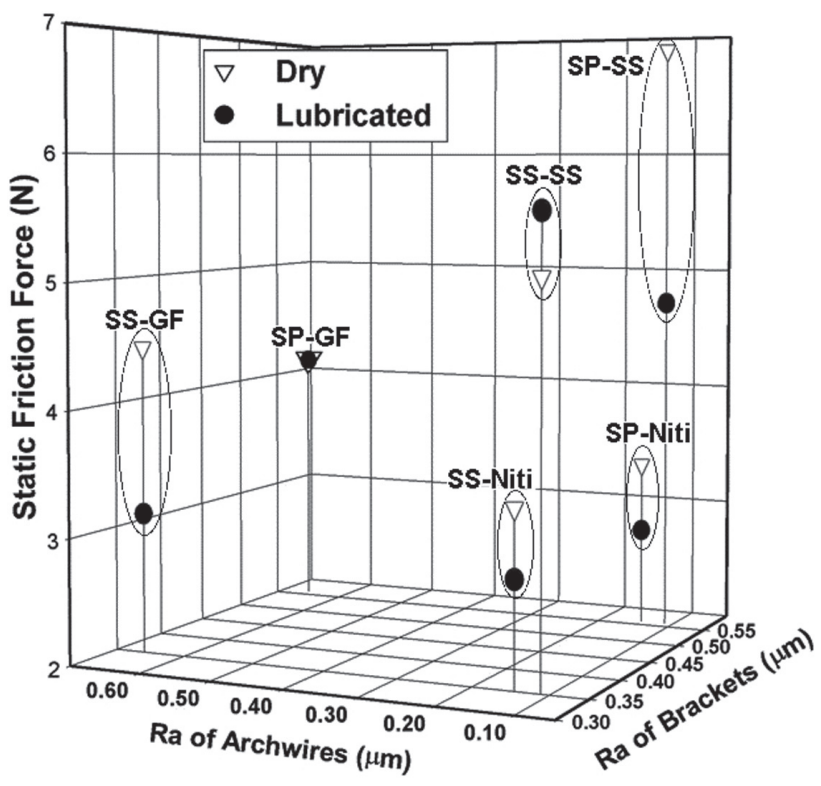

Fig. 4 3D mesh plot of static friction against roughness on dry and lubricated condition.

\section{DISCUSSION}

In the case of the brackets, the observation using SEM (Figs. 5a and 5b) reveals the size and shape of the slots which the rounded archwires slide through. This type of bracket is denominated edgewise and provides a better control over the tooth movements due to the curved and inclinable design to adapt to the tooth shape. Furthermore, the good surface finish of both brackets is clearly noticeable.

The SEM images of the archwires are shown in Figs. $5 \mathrm{c}, 5 \mathrm{~d}$ and 6 , where the differences in the quality of the surface are appreciable. In the case of GF archwire, the surface presents higher roughness and cracks (Figs. 6a and 6b) due to the presence of glass fibers at the surface, as can be confirmed by the $\mathrm{Si}$ elemental mapping (Figs. 6c and 6d). The roughness data confirm the SEM observation that the glass fibers at the surface provide a rough finish compared with the SS and Niti archwires.

In general, when friction data display negligible oscillation, the stick-slip phenomenon is absent, which would result in improved user comfort. In Figs. 2 and 3 , it can be seen that friction oscillations are small, particularly for the SS-SS contact (Fig. 3b).

If we focus initially on the nature of the brackets, it can be seen that for the SP bracket friction forces with the different archwires are similar under dry and lubricated configurations. Especially in the case of the SP-GF contact, the differences between dry and lubricated are minimal. This observation could be attributed to the failure of the saliva to effectively separate surfaces, generating an elastohydrodynamic lubrication regime. Probably, this low lubrication 

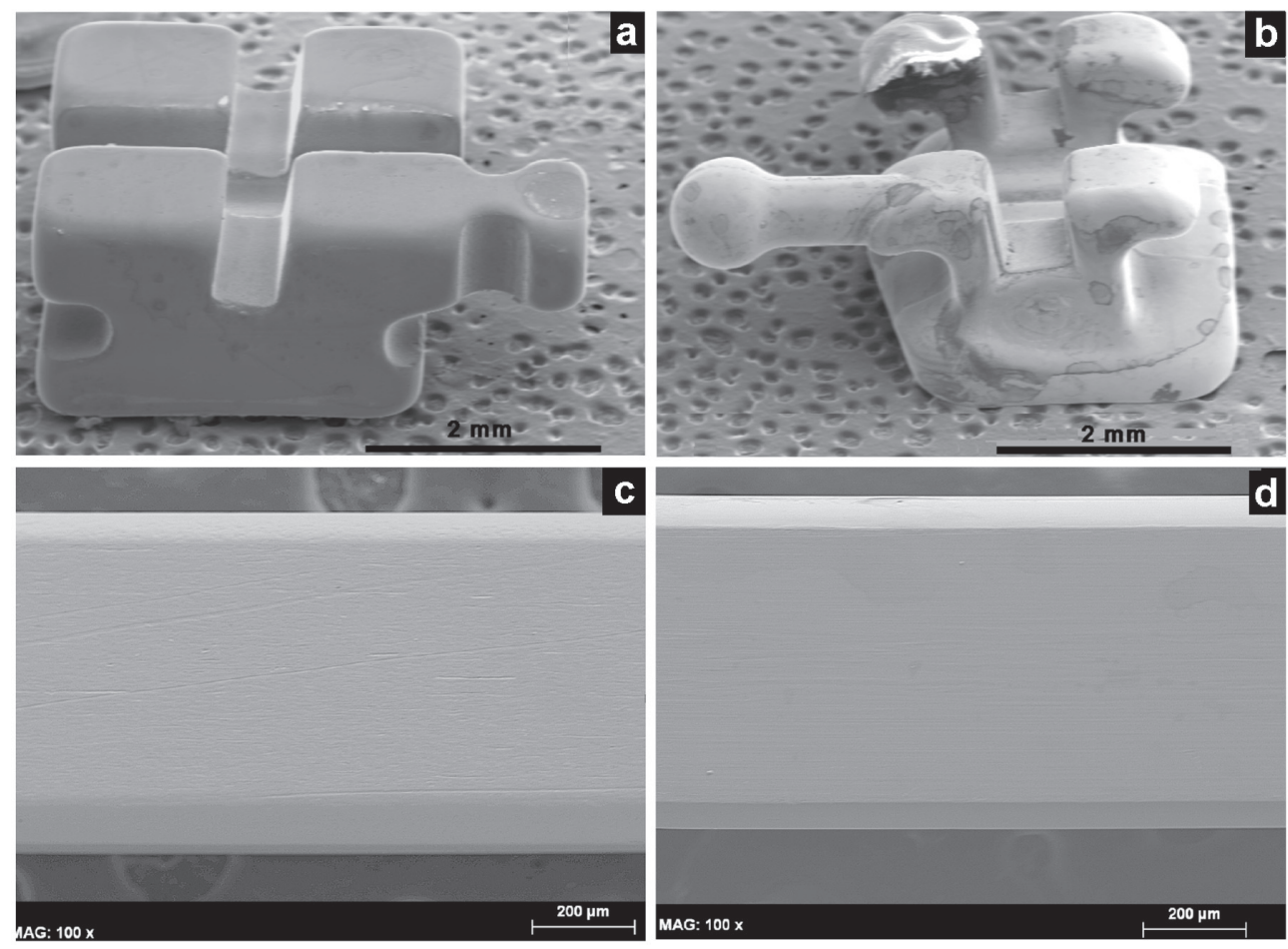

Fig. 5 SEM images of: a-Sapphire bracket, b-Stainless Steel bracket, c-Nitinol 60 archwire and d-stainless steel archwire.
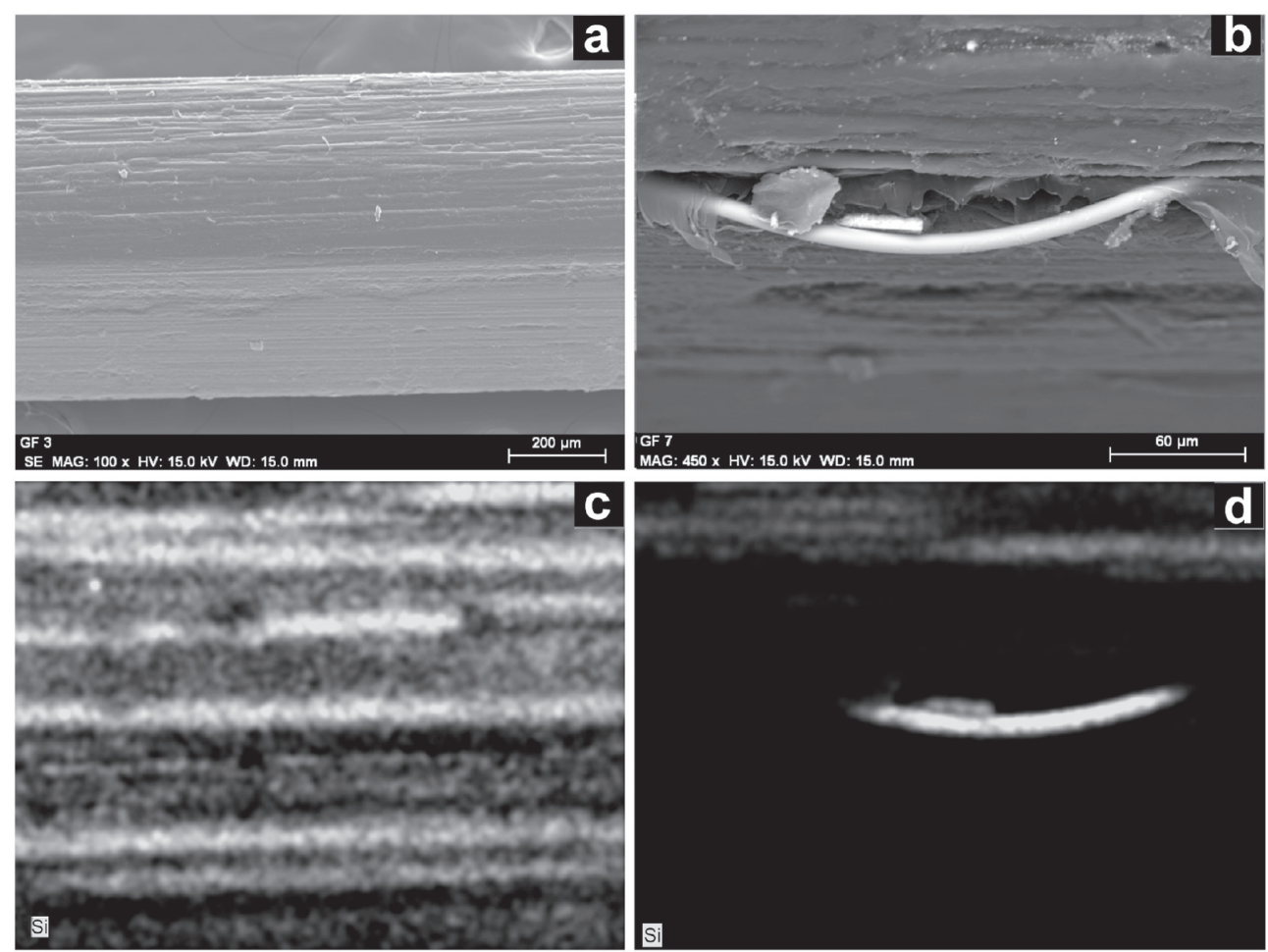

Fig. 6 SEM image of the glass fiber composite archwire.

a-Surface, b-Presence of a crack, c- and d-Elemental mappings of Silicon of 3a and 3b, respectively. 
performance is related to the high roughness value found in the GF surface.

With reference to the tests using the SS bracket, for both Niti and SS archwires, the lubricated configurations gave the highest values of friction forces. In these cases, the lubrication of the surface is not effective and there is in fact boundary contact between the materials. Of all twelve configurations tested the SS-SS pair shows the highest friction forces, both dry and lubricated.

The asperities of the surface of the steel make contact with the counterface regardless of the dry or lubricated regime or the nature of the brackets. The use of artificial saliva acts like an adhesive ${ }^{9)}$ and does not succeed in separating the surfaces.

This could be attributable to the shape memory properties and high thermal conductivity of this alloy, which would reduce the adherence to the SS surface of the bracket and would be more effective in dissipating the friction energy.

The SS-SS contact shows higher lubricated friction than dry friction, showing that lubrication is not only ineffective, but that the saliva fluid could cause corrosive interaction.

The best results are obtained for low roughness archwires, regardless of bracket roughness. The highest static friction values under dry contact are obtained for high roughness materials, either bracket or wire.

The high surface roughness and the defects present in the GF wire make lubrication ineffective against the sapphire bracket, showing the same friction value as under dry conditions. This could be explained by the difficulty in sliding the ceramic (GF)-ceramic (SP) surface asperities against each other due to the brittle nature of both materials.

\section{CONCLUSION}

Dry friction force values are higher than lubricated ones, except in the case of metal-metal contacts, where the corrosive interactions between artificial saliva and metal surfaces increase friction values.

The higher surface roughness of the glass-fiber reinforced composite wire could be responsible for the high static and kinetic friction forces, both under dry and lubricated conditions, particularly against the sapphire bracket, due to the higher roughness of sapphire with respect to stainless steel and to the existence of ceramic-ceramic sliding contacts of glass fiber against sapphire.
Although stainless steel against itself is the lowest surface roughness pair, the adhesive forces at the interface increase friction with respect to Nitinolstainless steel, which presents the lowest static and kinetic friction force values, both under dry and lubricated conditions. This result could be attributed to the low surface roughness and high corrosion resistance of the Nitinol wire.

\section{ACKNOWLEDGMENTS}

The authors wish to thank the financial support of the Ministerio de Economía y Competitividad (MINECO, Spain; FEDER, EU) (Grant MAT2014-55384-P). "Este trabajo es resultado de los proyectos de investigación (19292/PI/14,19544/GERM/14-19877/GERM/14) financiados por la Fundación Séneca-Agencia de Ciencia y Tecnología de la Región de Murcia en el marco del PCTIRM2011-2014".

\section{REFERENCES}

1) Rossouw PE. Friction: An overview. Semin Orthod 2003; 9: 218-222.

2) Wichelhaus A, Geserick M, Hibst R, Sander FG. The effect of surface treatment and clinical used on friction in NiTi orthodontic wires. Dent Mater 2005; 21: 938-945.

3) Yu JH, Huang HL, Wu LC, Hsu JT, Chang YY, Huang HH, Tsai MT. Friction of stainless steel nickel-titanium alloy and beta-titanium archwires in two commonly used orthodontic brackets. J Mech Biol 2011; 11: 917-928.

4) Yu JH, Wu LC, Hsu JT, Chang YY, Huang HH, Huang HL. Surface roughness and topography of four commonly used types of orthodontic archwire. J Med Biol Eng 2011; 31: 367 370.

5) Choi SH, Kang DY, Hwang CJ. Surface roughness of three types of modern plastic bracket slot floors and frictional resistance. Angle Orthod 2014; 84: 177-183.

6) Murayama M, Namura Y, Tamura T, Iwai H, Shimizu N. Relationship between friction force and orthodontic force at the leveling stage using a coated wire. J Appl Oral Sci 2013; 21: 554-559.

7) Chang CJ, Lee TM, Liu JK. Effect of bracket bevel design and oral environmental factors on frictional resistance. Angle Orthod 2013; 83: 956-965.

8) Fidalgo TK, Pithon MM, Maciel JV, Bolognese AM. Friction between different wire bracket combinations in artificial saliva-an in vitro evaluation. J Appl Oral Sci 2011; 19: 5762 .

9) Kusy RP, Whitley JQ. Resistance to sliding of orthodontic appliances in the dry and wet states: Influence of archwire alloy, interbracket distance, and bracket engagement. J Biomed Mater Res 2000; 52: 797-811. 